OPEN ACCESS

Edited by:

Thomas Grunewald,

Ludwig-Maximilians-Universität

München, Germany

Reviewed by:

Joanna Kitlinska,

Georgetown University, USA

Joseph A. Ludwig,

MD Anderson Cancer Center, USA

*Correspondence:

Françoise Redin

francoise.redini@univ-nantes.fr

Specialty section:

This article was submitted to

Pediatric Oncology,

a section of the journal

Frontiers in Oncology

Received: 08 June 2015 Accepted: 27 November 2015 Published: 23 December 2015

Citation:

Redini F and Heymann D (2015)

Bone Tumor Environment as a

Potential Therapeutic Target in Ewing

Sarcoma.

Front. Oncol. 5:279.

doi: $10.3389 /$ fonc.2015.00279

\section{Bone Tumor Environment as a Potential Therapeutic Target in Ewing Sarcoma}

\author{
Françoise Redini ${ }^{1,2,3 *}$ and Dominique Heymann ${ }^{1,2,3,4}$
}

${ }^{1}$ INSERM UMR_S 957, Nantes, France, ${ }^{2}$ Equipe labellisée Ligue contre le Cancer 2012, Nantes, France, ${ }^{3}$ Laboratoire de Physiopathologie de la Résorption osseuse et Thérapie des tumeurs osseuses primitives, Faculté de Médecine, Nantes, France, ${ }^{4} \mathrm{CHU}$ Hôtel-Dieu, Nantes, France

Ewing sarcoma is the second most common pediatric bone tumor, with three cases per million worldwide. In clinical terms, Ewing sarcoma is an aggressive, rapidly fatal malignancy that mainly develops not only in osseous sites (85\%) but also in extra-skeletal soft tissue. It spreads naturally to the lungs, bones, and bone marrow with poor prognosis in the two latter cases. Bone lesions from primary or secondary (metastases) tumors are characterized by extensive bone remodeling, more often due to osteolysis. Osteoclast activation and subsequent bone resorption are responsible for the clinical features of bone tumors, including pain, vertebral collapse, and spinal cord compression. Based on the "vicious cycle" concept of tumor cells and bone resorbing cells, drugs, which target osteoclasts, may be promising agents as adjuvant setting for treating bone tumors, including Ewing sarcoma. There is also increasing evidence that cellular and molecular protagonists present in the bone microenvironment play a part in establishing a favorable "niche" for tumor initiation and progression. The purpose of this review is to discuss the potential therapeutic value of drugs targeting the bone tumor microenvironment in Ewing sarcoma. The first part of the review will focus on targeting the bone resorbing function of osteoclasts by means of bisphosphonates or drugs blocking the pro-resorbing cytokine receptor activator of NF-kappa B ligand. Second, the role of this peculiar hypoxic microenvironment will be discussed in the context of resistance to chemotherapy, escape from the immune system, or neo-angiogenesis. Therapeutic interventions based on these specificities could be then proposed in the context of Ewing sarcoma.

Keywords: Ewing sarcoma, bone remodeling, bisphosphonate, RANKL, microenvironment, tumor bone niche, 3D models

\section{INTRODUCTION}

\section{Ewing Sarcoma: A Clinical Presentation}

Ewing sarcoma was first described by James Ewing in 1921. It is a high-grade neoplasm, and it is the second most common primary bone malignancy in both children and adolescents (1). With peak incidence at 15 years, this disease accounts for $2 \%$ of childhood cancers (2). Ewing sarcoma is defined as a bone tumor, which may occur at any site within the skeleton but preferentially affects the trunk and the diaphysis of long bones (2). However, it may occur in extra-skeletal soft tissue in $15 \%$ of cases. It is characterized by rapid tumor growth and extensive bone destruction (Figure 1) that can 


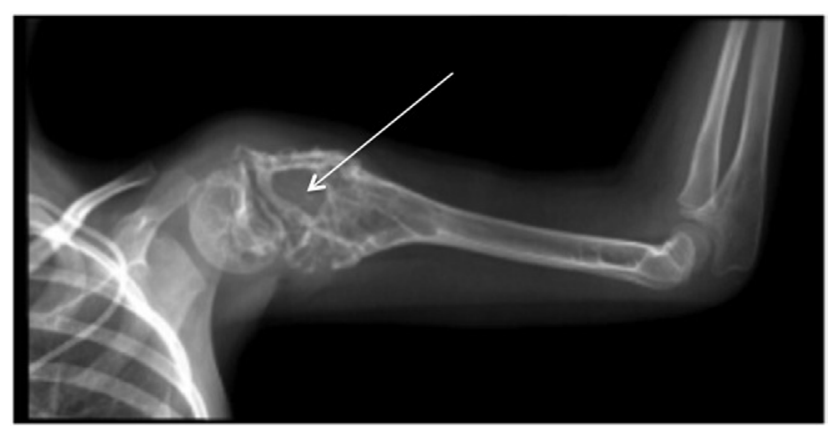

FIGURE 1 | X-ray of typical severe osteolytic lesions in a Ewing sarcoma patient (arrows: severe osteolytic lesions).

result in bone pain and pathological fracture (3). At the histological level, Ewing sarcoma appears as small, poorly differentiated, round tumor cells positive for the transmembrane glycoprotein CD99 staining (4).

The molecular event that initiates the Ewing's family of tumors is a typical chromosomal translocation that occurs in cells of mesenchymal origin and that fuses the EWS gene on chromosome $22 \mathrm{q} 12$ to a member of the erythroblast transformation sequence (ETS) transcription gene family, most commonly FLI-1, on 11q24 in $85 \%$ of cases (5-7). This translocation leads to the production of the oncogenic fusion gene EWS-FLI1, an aberrant transcription factor that promotes tumorigenicity $(8,9)$. The presence of this fusion gene, which represents the Ewing sarcoma signature, is used as a specific diagnostic marker of the Ewing's family of tumors thanks to fluorescence in situ hybridization and RT-qPCR (10). Numerous biological pathways, such as those involving insulin-like growth factor receptor (IGFR), platelet-derived growth factor receptor (PDGFR), vascular endothelial growth factor receptor (VEGFR), Sonic HedgeHog (SHH) pathway activation, Wnt, and transforming growth factor (TGF)- $\beta$ receptor II pathway inhibition, are modulated by EWS-FLI1 activity, leading to proliferation, angiogenesis, immune system escape, metastatic potential, and treatment resistance that contribute to the Ewing sarcoma malignant phenotype (11).

\section{Therapeutic Limits}

The on-going treatments for Ewing sarcoma patients are effective in more than $70 \%$ of patients with localized disease. They elicit clinical responses in patients with metastatic disease but are not curative due to acquired resistance. Before the 1970s, amputation was the main therapeutic option, with 5-year survival of $<20 \%$. The introduction of first radiation and then chemotherapy in the 70s has modified the prognostic significantly, with the 5 -year event-free survival rate for localized tumors at around $65 \%$, and the overall survival rate close to $75 \%$. However, the survival rates decrease to $15-25 \%$ when metastases are detected at diagnosis, or in patients presenting resistance to treatment or with relapsed disease. In the past three decades, conventional therapies seem to have attained a survival plateau for these metastatic patients (12).
Improved poly-chemotherapy has made it possible to limit surgery and salvage limb, but in about $20 \%$ of cases, bone sarcomas have already disseminated at the time of diagnosis. In most cases, the distant metastases are located in the lungs, followed by the skeleton. Although Ewing sarcoma patients with lung metastases have overall survival of $45 \%$ at 5 years, those with bone or bone marrow metastases have very poor prognosis, with $<25 \%$ overall survival at 5 years. In the past, when therapy was limited to local control (surgery), nearly all patients who initially appeared to have a localized tumor developed distant metastases (13). Ewing sarcoma thus needs to be considered as a systemic disease, requiring systemic treatment, i.e., combination chemotherapy, as a rule. However, systemic therapy can never replace definitive local control with surgery and/or radiotherapy. The therapy used for Ewing sarcoma therefore requires a combination of surgery or radiotherapy for localized control and high-intensity chemotherapy for localized and disseminated disease. The most recent protocol for Ewing tumors was the European Ewing tumor Working Initiative of National Groups 99 protocol (EuroEWING99, clinicaltrials. gov no. NCT00020566), which tested the benefits of a different chemotherapy combination involving vincristine, ifosfamide, doxorubicin, and etoposide (VIDE). The protocol was composed of six sequences of VIDE treatment followed by surgery when possible. The histological response to chemotherapy was then evaluated and patients were divided into three arms depending on the localization of the tumor at diagnosis, the volume for unresected tumors, and the percentage of residual cells after treatment. The R1 arm included patients with localized disease and a good response to chemotherapy ( $<10 \%$ of residual cells) or with a volume of $<200 \mathrm{ml}$. The R2 arm included patients with lung metastases and patients with localized tumors and a poor response to chemotherapy, or with a volume of more than $200 \mathrm{ml}$. Finally, the R3 arm included patients with bone, bone marrow, or multifocal metastases. The current survival rate for EuroEWING patients has attained $80 \%$ for localized disease of small volume (R1). Unfortunately, the 5-year survival rate for patients with metastases detected at diagnosis remains around 25\% and even around $10 \%$ when relapse occurs within the first 2 years following treatment.

The current protocol for Ewing sarcoma patients is the EuroEWING2012 (clinicaltrials.gov no. NCT00987636), which started in December 2014 in Great Britain, with two randomizations: the first compares two chemotherapy protocols (with surgery and/or radiotherapy) and the second randomizes patients with or without bisphosphonate zoledronate (zometa ${ }^{\circledR}$ ).

Given that survival rates had not evolved in more than three decades, especially for metastatic patients with a very poor initial prognosis, there was an urgent need to define new therapeutic targets for Ewing sarcoma patients. In addition to the tumor cells themselves, targeting the bone tumor microenvironment appears to be promising.

\section{The Bone Microenvironment Is a Favorable "Niche" for Tumor Progression in Bone}

Recently, there has been a dramatic increase in the importance given to the theory that the bone microenvironment participates 
in determining the "bone niche" in the progression of bone tumors, and in establishing resistance processes to conventional therapies. The concept of "bone niche" is well-recognized in the context of hematological malignancies, such as leukemia (14) or multiple myeloma (15). The "niche" is a functional microenvironment able to both promote the emergence of cancer stem cells and provide all factors required for their development. However, the bone niche is composed of numerous cell types (pre-osteoclasts, pre-osteoblasts, endothelial cells, macrophages, etc.) that are located in the bone matrix, and their functional coordination is a pre-requisite for maintaining the bone and bone niche microarchitecture.

Much research has been published on the role played by the bone microenvironment in establishing metastases in these organs, especially from breast or prostate carcinomas. The concept of bone niche is also currently under discussion in the case of solid tumors, and strengthens the "seed and soil" theory proposed by Paget in 1887, in which tumor cells ("seeds") colonize receptive foci ("soil") (16). These data are supported by the fact that specific molecules (such as cadherin and osteopontin) play a part in stabilizing cancer cells in bone niches, mimicking the cell interactions that take place during hemopoiesis, as identified in the pre-metastatic niche in breast carcinoma $(17,18)$. In addition, carcinoma cells grow well in bone, which stores a variety of cytokines and growth factors, and thus provides an extremely fertile environment for growing tumor cells $(19,20)$.

The "seed and soil" theory can be also envisaged for primary bone tumors, as tumor growth and metastasis often require constant interactions between tumor cells and their surrounding microenvironment (21-25). This hypothesis has been largely documented in the case of osteosarcoma $(26,27)$ and chondrosarcoma (28), but very little information is currently available for Ewing sarcoma.

\section{The Concept of the Vicious Cycle in Ewing Sarcoma}

Ewing sarcoma is characterized by extensive bone destruction, mainly due to osteolysis (Figure 1). Because Ewing sarcoma cells cannot directly degrade bone, osteoclast activation and subsequent bone resorption may be responsible for the clinical features of bone destruction in this pathology (3). Bone degradation is controlled by osteoclasts, whose differentiation and activation are mainly mediated by receptor activator of NF-kappa B ligand (RANKL), a member of the tumor necrosis factor (TNF) superfamily (TNFSF11) after it binds to its receptor RANK expressed at the surface of mature osteoclasts and osteoclast precursors (29) (Figure 2). Osteoprotegerin (OPG) acts as a decoy receptor inhibiting osteoclast formation, function, and survival by preventing the binding of RANKL to its receptor RANK (26).

Interaction between tumor cells, tumor-derived humoral factors, and the bone marrow in the bone niche has been shown to be essential for bone tumor initiation and promotion (30, 31). Targeting the bone microenvironment, and particularly osteoclast activation, may therefore be a promising adjuvant strategy for treating bone tumors, including Ewing sarcoma. The vicious cycle between osteoclasts, bone stromal cells/osteoblasts, and cancer cells has been hypothesized during the progression of primary bone tumors (32) (Figure 2). Tumor cells produce osteoclast activating factors, such as interleukin (IL)-6, TNF- $\alpha$, or ParaThyroid Hormone-related Peptide (PTH-rP), which induce osteoclast differentiation and activation. When osteoclasts resorb bone, they allow the release of growth factors stored in the bone matrix (TGF- $\beta$, IGF-1, PDGF, etc.), which in turn activate tumor cell proliferation (32). Accordingly, inhibiting osteoclast activity is a promising approach for breaking the vicious cycle, and thus indirectly limiting local cancer growth.

In addition, new therapeutic options targeting hypoxia, angiogenesis, bone cells, or mediators in the particular bone microenvironment have been studied extensively at the preclinical level, with the more promising now being proposed in clinical trials. This review will describe the most recent developments in such therapeutic options for Ewing sarcoma patients.

\section{TARGETING BONE CELLS IN EWING SARCOMA}

Therapeutic agents that target the bone environment and modulate bone metabolism have been studied in preclinical models of primary bone sarcomas, demonstrating a certain degree of efficacy in both osteosarcoma and Ewing sarcoma. Two main strategies are currently being developed: (i) the first directly targets osteoclasts (differentiation, activation, and functions), mainly using bisphosphonates (BPs), and (ii) the second targets the cytokine RANKL, the pivotal cytokine for regulating osteoclast activation.

\section{Bisphosphonates}

Bisphosphonates are the synthetic analogs of endogenous pyrophosphate, with a high resistance to protease degradation, and the ability to strongly inhibit bone resorption (33). They are composed of two phosphonate groups. The central oxygen atom in pyrophosphate is replaced by a carbon atom, which allows the substitution of two side groups, one of which is often an hydroxyl group, and the other defines the BP generation (Figure 3). Two main families can therefore be distinguished: nitrogen- and non-nitrogen-containing BPs, which act on osteoclasts by means of different molecular mechanisms. In both cases, the final result - common to both - is the induction of osteoclast apoptosis. BPs act either by inhibiting the recruitment, proliferation, and differentiation of pre-osteoclasts or by impeding the resorptive activity of mature osteoclasts (34-37). Zoledronic acid (ZOL) belongs to the third generation of BPs, which is the most efficient for preventing bone lesions (38-40). As for other nitrogencontaining BPs, ZOL inhibits the farnesyl diphosphate and geranylgeranyl diphosphate synthases, two enzymes involved in the mevalonate pathway necessary for the prenylation of small intracellular GTPases, such as Ras, Rho, or Rac (41). As the prenylation of these GTPases is essential for osteoclast function, their inhibition leads to osteoclast apoptosis as a result of the loss of the survival signal (42-44). Moreover, BPs may also inhibit bone resorption by increasing the production of OPG by human osteoblasts (45). OPG is the decoy receptor of RANKL, 


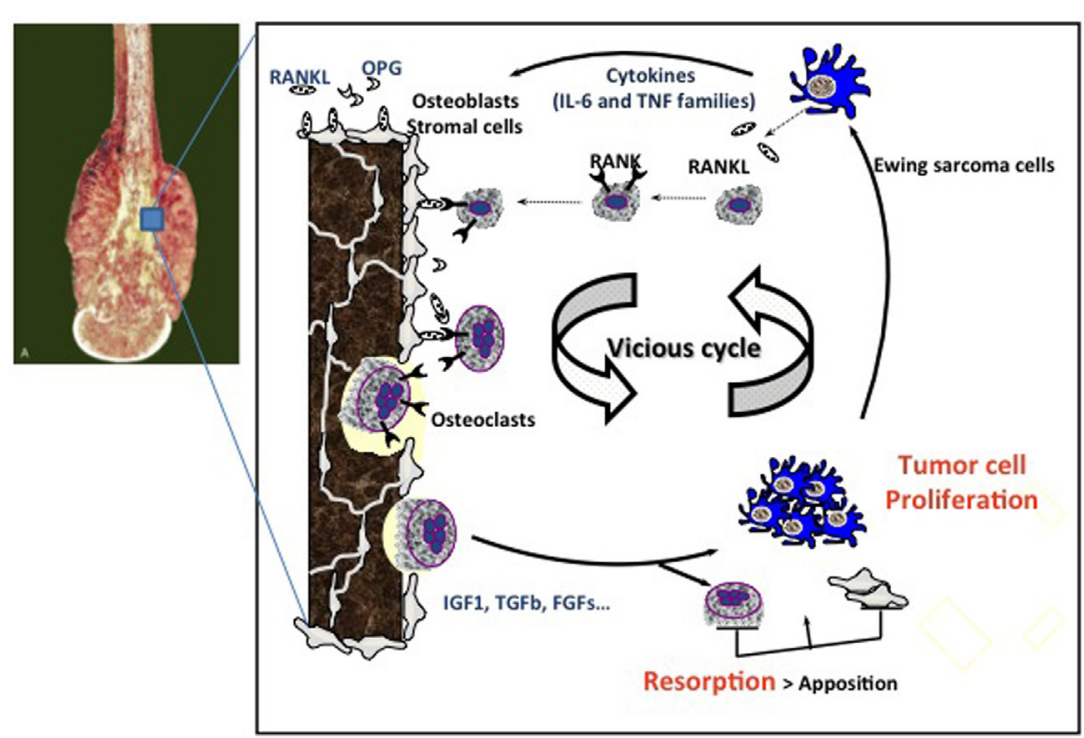

FIGURE 2 | Vicious cycle between Ewing sarcoma cell proliferation and osteoclast activation. Tumor cells produce osteoclast activating factors (IL-6, $\mathrm{TNF}-\alpha$, etc.) that will induce osteoclast differentiation and activation. When they resorb bone, osteoclasts allow the release of growth factors stored in the bone matrix, such as IGF-1, FGFs, and TGF- $\beta$, which in turn activate tumor cell proliferation. This is the theory of the so-called "vicious cycle." The molecular OPG/ RANKL/RANK triad plays a pivotal role in the regulation of bone resorption. OPG and RANKL are produced by osteoblasts and/or stromal cells, whereas RANK is expressed at the surface of osteoclasts and their precursors. OPG, osteoprotegerin; IL-6, interleukin-6; TNF, tumor necrosis factor; RANK, receptor activator of NF-kB; RANKL, RANK-ligand; IGF1, insulin-like growth factor1; TGF- $\beta$, transforming growth factor- $\beta$; FGFs, fibroblast growth factors.

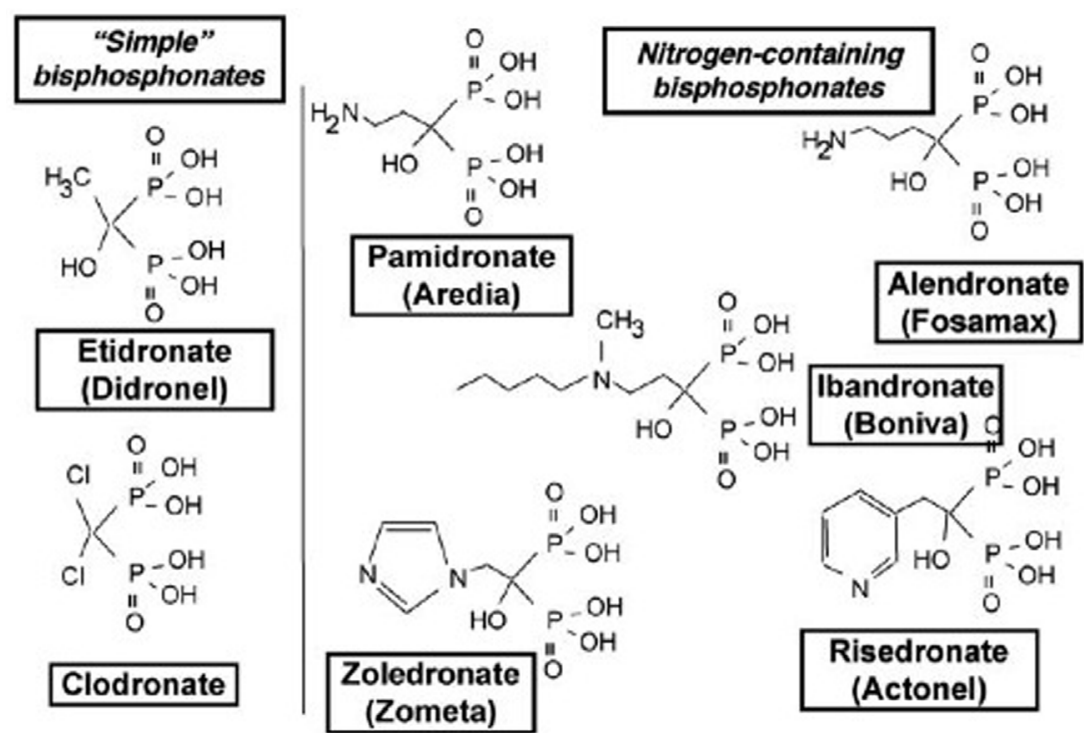

FIGURE 3 | Schematic representation of the different bisphosphonate (BP) families: simple non-N-containing BPs (etidronate and clodronate) and $\mathrm{N}$-containing BPs (pamidronate, alendronate, idandronate, risedronate, and zoledronate).

which inhibits the RANK/RANKL interaction that is essential for osteoclast differentiation and activation. In addition to the antiresorptive effect of ZOL, it has been shown to induce the death of tumor cell lines, such as myeloma, and breast and prostate carcinoma cells in several preclinical studies (39). It also appears to exert an inhibitory effect on cancer cell invasion and angiogenesis (46).

Despite several side effects reported after long-term treatment with BPs, including osteonecrosis of the jaw, BPs are currently under investigation in postmenopausal bone loss and bone 
lesions of tumoral origin, such as bone metastases from breast and prostate cancer with variable clinical benefits $(47,48)$. A significant decrease in bone resorption was observed in these studies but with no unequivocal effects on survival or the occurrence of metastases. The clinical effects of BPs on bone metastases from lung cancer are also discussed, as are their effects on visceral metastases (49-52). The encouraging results reported on bone remodeling, as well as the ability of BPs, and in particular ZOL, to induce tumor cell death in vitro, make them good candidates for a therapeutic strategy in primary bone tumors. ZOL may effectively inhibit both bone resorption and tumor proliferation in the vicious cycle, making it more efficient. With regard to primary bone tumors, several studies have already demonstrated the benefits of using ZOL in osteosarcoma (53-56), in particular, promising preclinical results have been reported on survival and tumor growth (56). In this context, ZOL has recently been combined with conventional chemotherapy and surgery for adult and pediatric patients in the French OS2006 phase III randomized clinical trial for osteosarcoma treatment. Following these results, other preclinical and clinical studies demonstrated the beneficial effect of BP treatment in osteosarcoma (57-61).

In Ewing sarcoma, despite improvements to chemotherapy protocols, the survival rate for patients with bone metastases remains very low. In this context, combining $\mathrm{ZOL}$ with current conventional chemotherapy may be a promising therapeutic option for both limiting tumor-associated osteolysis and preventing the development of bone metastases, which is currently the main factor for a bad prognosis for this pathology (62). Few fundamental and preclinical studies have demonstrated an antitumoral effect for ZOL on Ewing sarcoma cell lines $(63,64)$. Of these studies, our team has recently shown that ZOL significantly inhibits tumor cell viability by blocking the cell cycle in $S-G_{2} M$ phase transition and by promoting caspase-3 activation (65). Using preclinical models of Ewing sarcoma induced in athymic mice by injecting human Ewing sarcoma cells either in bone site or in soft tissue, ZOL alone significantly inhibited tumor development in bone sites, decreasing osteolytic lesions and improving mouse survival (65). On the contrary, the same doses of ZOL had no effect on Ewing sarcoma progression in soft tissue. These results can be explained by the high tropism of BPs for the calcified bone matrix, leading to their elevated concentration in bone tissue and their rapid clearance from blood and soft tissue. These data correlate with other studies on soft tissue tumors or visceral metastases (39). On the other hand, we demonstrated the synergistic effect of a combination of ZOL and ifosfamide, a conventional drug used in Ewing sarcoma clinical protocols, on tumor progression in soft tissue (65). These results correlate with previous studies showing a synergistic effect between BPs and chemotherapeutical agents and demonstrate the great benefit of using ZOL in Ewing sarcoma treatments as a means of reducing the chemotherapy doses and as a consequence, their side effects (58, 66-68).

With regard to invasion and migration, we have already published that treatment with ZOL inhibits Ewing sarcoma cell migration in vitro in Boyden chambers and diminishes MMP-2 activity as revealed by zymography (69). In addition, less pulmonary metastases were observed in mice treated with
ZOL compared to untreated animals, in a model of spontaneous metastases disseminated from primary Ewing sarcoma induced in bone (69).

For the transfer to clinical practice, one phase II study evaluating the combination of chemotherapy and pamidronate in osteosarcoma patients has demonstrated little impact on patient survival, but has been shown to improve the durability of limb reconstruction (61). In a recently completed phase I study, ZOL combined with conventional multi-drug chemotherapy was safe, but failed to reveal any significant differences in event-free or overall survival in patients with newly diagnosed metastatic osteosarcoma (70). There are three phase II/III trials currently in progress, evaluating the efficacy of ZOL as a single agent or an adjuvant to chemotherapy in localized and metastatic osteosarcoma (NCT00691236 and NCT00470223) and in Ewing sarcoma (NCT00987636).

However, long-term use of BPs may impact bone growth and tooth eruption in young patients. In our laboratory, we have carried out preclinical studies on newborn mice treated or not with ZOL, using a protocol that reproduces the frequency and doses administered in humans. ZOL induces a reversible arrest in bone growth that was also observed in young patients treated with zometa ${ }^{\circledR}(71)$. For tooth eruption, irreversible inhibition was observed (72).

As several side effects have been reported with the clinical use of BPs $(49,50)$, another approach to decrease bone resorption could therefore be to target RANKL, the main cytokine involved in osteoclast differentiation.

\section{Anti-RANKL Strategies}

Bone remodeling is strongly regulated thanks to the molecular triad OPG-RANKL-RANK (26). The binding of RANKL to its receptor RANK, expressed on the surface of osteoclast precursors, induces osteoclast differentiation in vitro in addition to macrophage-colony stimulating factor (M-CSF), suggesting that this differentiation plays an important role in bone biology. In the bone microenvironment, RANKL is produced by bone marrow stromal cells and osteoblasts, while in a bone tumor environment, it can be produced by other cell types, such as fibroblasts, epithelial cells, or T-lymphocytes, in which RANKL appears to be the final effector of osteoclast-mediated bone resorption (26). Cells from many tumor types, including multiple myeloma, prostate cancer, or even human neuroblastoma, can also express RANKL themselves (73-75). Moreover, many of the chemokines, cytokines, hormones, and growth factors produced by tumor cells are able to induce an increase in RANKL expression through $\mathrm{PTH}-\mathrm{rP}$, and a decrease in OPG production, thus aggravating the vicious cycle in bone metastases. RANK is one of the signaling molecules associated with worse outcomes in osteosarcoma. High expression of RANKL is associated with reduced survival in osteosarcoma, and it has been reported that osteosarcoma cell lines and biopsies show high expression of functional RANK, suggesting a potential autocrine stimulation of this pathway (76, 77). Inhibition of RANKL using the shRNA strategy reduced motility and anoikis resistance in osteosarcoma cell lines, whereas overexpression of RANK increased OS cell motility without affecting cell proliferation (78). One study reported the 
preventive effect of siRNA-RANKL on tumor progression when associated with the chemotherapeutic agent ifosfamide in a preclinical model of osteosarcoma (79). For Ewing sarcoma, only a few studies are available, but it seems that these cells express only a low level of RANKL (3). In our case, preliminary preclinical studies evidenced localized but strong expression of RANKL in a paratibial model of Ewing sarcoma induced by an intramuscular injection of human A673 cells in Nude mice (80). The advantages of targeting RANKL have previously been reported in both bone metastases and primary bone tumors and might be a promising target in Ewing sarcoma (73, 74, 81, 82). Several molecules targeting RANKL have already proved their efficiency in other malignant bone pathologies, such as osteosarcoma, and might be a potent therapeutic agent in Ewing sarcoma.

Osteoprotegerin, a member of the TNF receptor super-family, is a ubiquitous secreted homodimeric cytokine able to bind RANKL and then inhibit the RANK/RANKL interaction, as well as any further osteoclast differentiation and activation (83-85). A disruption in the RANKL/OPG ratio in favor of RANKL has been shown to be responsible for severe osteolysis in a tumoral context (86). Accordingly, overexpressing OPG to restore this equilibrium between OPG and RANKL expression appears to be a promising approach for limiting tumor-associated bone lesions. For the first time, our team has shown significant therapeutic benefits of OPG in primary bone tumors. In a preclinical model of osteosarcoma, OPG delivered by non-viral gene transfer effectively inhibited tumor growth and tumor-associated osteolysis, significantly increasing animal survival (81). Several studies have tested OPG overexpression in OS and Ewing sarcoma preclinical models with promising results, especially in osteosarcoma (81). Moreover, despite its clinical efficiency in preventing osteolytic lesions, a major issue for OPG-Fc administration as an adjuvant therapeutic agent in a tumor context is its ability to inhibit the apoptosis induced by TNF-related apoptosis inducing ligand (TRAIL) (87). The dual effect of OPG may inhibit TRAILinduced apoptosis of tumor cells, a natural mechanism for preventing tumor development (88). In addition, TRAIL's ability to both induce apoptosis in sensitive Ewing Sarcoma cell lines and prevent tumor development has already been demonstrated in vitro by Wietzerbin's team and in vivo by our team in a preclinical model induced by intratibial injection of Ewing sarcoma cells in nude mice $(89,90)$. To avoid the potential protumoral effect of OPG, the recombinant protein RANK-Fc, the soluble form of RANK, could be used in Ewing sarcoma to block RANKL activity. RANK-Fc is unable to bind TRAIL, and its efficacy has already been demonstrated in preventing tumor-associated osteolysis and, indirectly, tumor growth in preclinical models of bone metastases, such as prostate, lung, and breast cancer (91-93). Our team also showed how RANK-Fc, when delivered by non-viral gene transfer, is able to prevent osteolytic lesions and tumor development, thus inducing an increase in animal survival in a preclinical rodent model of osteosarcoma (82). The same efficacy can be expected in Ewing sarcoma but remains to be tested. For clinical transfer, denosumab is a monoclonal antibody specific for human RANKL, which was initially developed to treat osteoporosis (94). It was then used for painful bone metastases with effective results (95-98). It was subsequently found to also be effective for giant cell tumor of bone, a benign but destructive neoplasm with severe osteolytic lesions, in which transformed mononuclear cells secrete high levels of RANKL, causing osteoclast hyperactivity (99).

\section{TARGETING OTHER ASPECTS OF THE BONE MICROENVIRONMENT IN EWING SARCOMA}

Besides bone cells themselves, the tumor microenvironment of primary bone tumors provides factors that are favorable for tumor initiation, progression, therapy resistance, or metastatic dissemination. Of the different constituents or aspects of this peculiar microenvironment, special attention has been paid to hypoxia, escape from the immune system, angiogenesis, growth factors from the microenvironment, and modification of the microenvironment itself by therapeutic agents that may interfere with tumor progression (Figure 4).

Hypoxia is an important condition in the tumor cell microenvironment associated with a more aggressive phenotype and poor prognosis of many cancers in adults. For example, intratumoral hypoxia is a common finding in breast cancer associated with a significantly increased risk of metastasis and patient mortality (100). Hypoxia-inducible factors activate the transcription of a large battery of genes encoding proteins that promote primary tumor vascularization and growth, stromal cell recruitment, extracellular matrix remodeling, pre-metastatic niche formation, cell motility, local tissue invasion, extravasation at sites of metastasis, and maintenance of the cancer stem cell phenotype that is required to generate secondary tumors. It is also known that severe and long-lasting hypoxia results in necrosis, thus being correlated with unfavorable outcome. Concerning Ewing sarcoma, a clinical study previously reported a strong correlation between the presence and the amount of necrotic areas in the tumor with the risk of metastases (101). In addition, Aryee et al. reported that HIF- $1 \alpha$ expression was detectable in $18 / 28$ primary tumors from the Ewing sarcoma family and that EWS-FLI1 was up-regulated in a HIF-1 $\alpha$-dependent manner (102). In addition, this study revealed that hypoxia stimulated the invasiveness and soft agar colony formation of Ewing sarcoma cells in vitro. Further studies suggest that EWS-FLI1 regulation in an hypoxic environment may occur at the posttranscriptional level, which is supported by the observation that HIF- $1 \alpha$-activated genes, such as VEGF, Aldolase-C, GLUT-1, CA9, and IGFBP3, were increased under hypoxia, whereas EWS-FLI1 RNA expression remained unchanged (103). It is also suggested that hypoxia increases Ewing sarcoma malignancy through enhancing invasive and colony-formation capacities. Furthermore, it could be proposed that hypoxia may contribute to the aggressive metastatic behavior of Ewing sarcoma, as HIF-1 $\alpha$ and EWS-FLI1 may function together in both synergistic and antagonistic cross-talk under hypoxia conditions. Therefore, drugs that target hypoxia need to be tested in Ewing sarcoma models.

Crosstalk between the bone niche and the immune system, known as "osteoimmunology," has been suggested as being a potential target for bone tumor treatment. There is a well-recognized link 


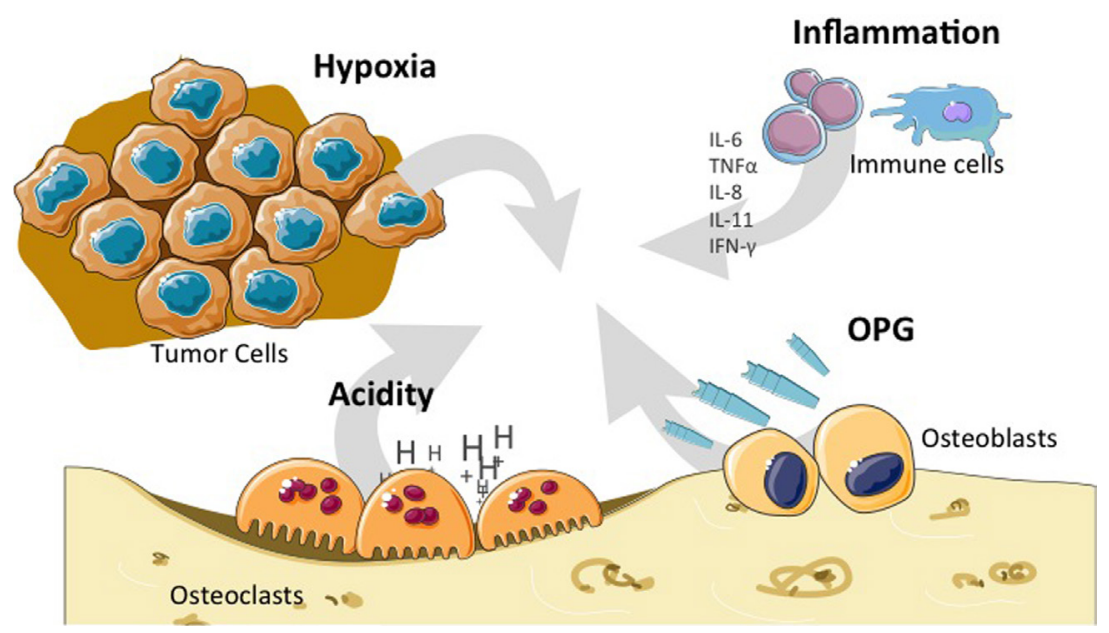

FIGURE 4 | Particular bone microenvironment that may affect tumor initiation, progression, or dissemination. Bone microenvironment is characterized by high hypoxia, high acidity, OPG release, and the influence of the cytokines (IL-6, IL-8, IL-11, and TNF- $\alpha$ ) produced by cells from the immune system. IL, interleukin; TNF, tumor necrosis factor; OPG, osteoprotegerin.

between bone constituents and the immune system, leading to recent efforts to elucidate the functions of molecules expressed in both bone and immune cells. A recent review nicely describes the complexity of the interaction between the skeletal and immune systems, suggesting that their interdependency needs to be taken into consideration when designing therapeutic approaches for either of the two systems (31). For example, denosumab, which was originally used to specifically target bone resorption, is now under evaluation for its effect on the long-term immune response. As both the bone and immune systems are often disrupted in cancer, they may be crucial in regulating tumor growth and progression. Certain therapies, such as BPs and RANKL-targeted drugs that aim to reduce pathological osteolysis in cancer, may interact with the immune system, thus providing favorable effects on survival. Another interesting publication reported that dynamic tumor-host immune interactions within the tumor microenvironment may polarize immune responses in situ, influencing tumor development and/or progression (104). They studied the nature of tumor-host immune interactions within the Ewing sarcoma microenvironment, analyzing the presence and spatial distribution of infiltrating $\mathrm{CD} 8(+)(/) \mathrm{CD} 4+$ T-lymphocytes in therapy-naive Ewing sarcoma. They observed that tumorinfiltrating T-cells contained significantly higher percentages of CD8(+) T-lymphocytes than stroma-infiltrating cells, suggesting preferential migration of this type of T-cell into tumor areas. Their results indicated that an inflammatory immune microenvironment with high expression of type 1-associated chemokines may be critical for the recruitment of CD8(+) T-lymphocytes expressing the corresponding chemokine receptors. The observed impact of tumor-infiltrating CD8(+) T-lymphocytes is consistent with there being a role for adaptive anti-tumor immunity in preventing Ewing sarcoma from progressing. Recognizing the merits and exploitation/induction of an inflammatory microenvironment may thus improve the efficacy of natural responses against, and (adoptive) immunotherapeutic approaches for, Ewing sarcoma.
With regard to angiogenesis, VEGF-165 expression in the tumor microenvironment has been shown to influence the differentiation of bone marrow-derived pericytes, which play a part in the vasculature of Ewing sarcoma (105). One year later, the same team demonstrated that VEGF-165 contributed to the osteolytic process in Ewing sarcoma by upregulating RANKL (106). They showed that VEGF-165, together with EWS-FLI1, increased RANKL promoter activity. This increase in RANKL gene expression in the bone marrow microenvironment during the metastatic process may be involved in tumor-induced bone osteolysis.

Other growth factors present in the bone microenvironment, such as basic FGF, may play a part in tumor progression as they enhance cell motility and invasion of the Ewing sarcoma family of tumors by activating the FGFR1-PI3K-Rac1 pathway (107). The authors therefore conclude that the bFGF-FGFR1-PI3K-Rac1 pathway in the bone microenvironment may have a significant role in the invasion and metastasis of the Ewing sarcoma family of tumors.

Conversely, therapeutic agents, such as ZOL, are able to modify the bonemicroenvironment surrounding primaryor disseminated tumor cells, as has been reported in breast cancer recurrence in bone (108). Treatment of mice with ZOL induced a rapid increase in trabecular bone volume versus controls, which was reflected by a significant reduction in osteoclast and osteoblast numbers per millimeter in trabecular bone, and reduced bone marker levels in serum. Pre-treatment with ZOL caused an accumulation of extracellular matrix in the growth plate associated with a trend for preferential homing of tumor cells to osteoblast-rich areas of bone, but without affecting the total number of tumor cells. The number of circulating tumor cells was reduced in ZOL-treated animals. Although this study concerns breast cancer, osteoblasts may be key components in the bone metastasis/tumor niche, and therefore a potential therapeutic target, at least in breast cancer. This hypothesis therefore needs to be studied extensively in primary bone tumors, including Ewing sarcoma. 


\section{BONE MICROENVIRONMENT MODELING IN EWING SARCOMA}

As the microenvironment, and especially the bone tumor microenvironment, can both inhibit and facilitate tumor growth and metastatic dissemination, better modelization of the tumor bone niche is needed to characterize tumor cell-stroma interaction in depth. It has been shown that osteoblasts, osteoclasts, fibroblasts, myeloid cells, and mesenchymal stem cells (MSCs) play essential roles in primary tumor growth and metastasis $(109,110)$. However, current in vitro approaches are far from replicating the native in vivo milieu in which tumors develop, a necessary condition for advancing cancer research and translating new therapies into clinical practice. Most preclinical anti-neoplastic drug testing is still carried out on conventional $2 \mathrm{D}$ cell culture systems. Although these systems mimic some of the phenotypic traits observed clinically, they are limited in their ability to model the full range of microenvironmental interactions, such as $3 \mathrm{D}$ cell-cell and cell-extracellular matrix interactions. Several teams have thus established ex vivo 3D bone tumor models that closely mimic the morphology, growth kinetics, and protein expression profile of human tumors, including Ewing sarcoma (111-113). For example, Ewing sarcoma cells cultured in porous 3D electrospun poly( $\epsilon$-caprolactone) scaffolds were not only more resistant to traditional cytotoxic drugs than cells in $2 \mathrm{D}$ monolayer cultures but also exhibited remarkable differences in the expression pattern of the IGF-1R/mTOR pathway (111). This 3D model of the bone microenvironment may therefore have broad applicability for mechanical studies of bone sarcomas and shows the potential for increasing preclinical evaluation of anti-neoplastic drug candidates for these malignancies. In the same way, Villasante et al. described a bioengineered model of human Ewing sarcoma that mimics the native bone tumor niche with high biological fidelity (113). In this model, cancer cells that have lost their transcriptional profiles after monolayer culture reexpress genes related to focal adhesion and cancer pathways. The bioengineered model recovers the original hypoxic and glycolytic tumor phenotype and makes possible re-expression of angiogenic and vasculogenic mimicry features that favor tumor adaptation. Differentially expressed genes between the monolayer cell culture and native tumor environment may thus be potential therapeutic targets that could be explored using the bioengineered tumor model.

In addition, Ludwig's team has highlighted a number of innovative methods used to fabricate biomimetic Ewing sarcoma, including both the surrounding cellular milieu and the extracellular matrix. These methods suggest potential applications for advancing our understanding of the biology of Ewing sarcoma, preclinical drug testing, and personalized medicine (112).

Finally, it appears that the bone microenvironment should be modelized in order to analyze the response of bone tumor cells to drug screening under optimal conditions. Currently, few preclinical models of bone cancer, and particularly Ewing sarcoma, mirror the site of the disease in patients, as they are mostly subcutaneous or intramuscular xenografts (114).

For metastasis in Ewing sarcoma, intravenous models induced in non-obese diabetic/severe combined immunodeficient (NSG) mice showed a pattern of disease spread similar to that found in patients, but only $23 \%$ of the experimental mice developed assessable bone metastases (115). It is therefore preferable to develop orthotopic models that involve direct injection of Ewing sarcoma cells at the clinically relevant site, i.e., intrafemoral. This type of injection in immunocompromized mice provides a technically feasible and reproducible approach, resulting in tumors that are detectable by palpation or in vivo imaging, and that closely resemble those observed in patients (116). The importance of such orthotopic models for testing potential new drugs at the preclinical level was emphasized in the study by Odri et al. (65), comparing how tumor progression responds to ZOL in two models of Ewing sarcoma: one induced by tumor cell injection in the medullar cavity of tibia and the other with initial progression in soft tissue (65). ZOL significantly inhibited Ewing sarcoma cell progression only in the intratibial model and showed no effect in the soft tissue. These results strongly suggest the importance of considering the complete bone microenvironment when testing new drugs, especially in the case of bone tumors, such as Ewing sarcoma. Recently, Vormoor et al. also developed an interesting preclinical orthotopic model of Ewing sarcoma in NSG mice, reproducing the biology of the tumor-bone interactions observed in human disease (117). In this model, the Ewing sarcoma cells have been modified allowing in vivo monitoring of disease progression (115). The authors therefore demonstrated the utility of small animal bioimaging for tracking disease progression, making this model a useful assay for preclinical drug testing.

\section{CONCLUSION - PERSPECTIVES}

Despite improvements in poly-chemotherapy combinations and surgical approaches preserving limbs from amputation, one group of Ewing sarcoma patients still remains at high risk, with poor survival rates. These patients present with metastatic disease at diagnosis or respond poorly to chemotherapy due to acquired resistance. New therapeutic options are thus needed. Given the growing interest in the microenvironment and its recognized involvement in cancer initiation and progression, it is relevant to propose therapeutic strategies that target molecular and/or cellular protagonists of the bone tumor microenvironment in the case of Ewing sarcoma.

Most on-going studies focus on bone cells, especially osteoclasts, either by directly targeting them or inhibiting RANKL, the main cytokine involved in osteoclast activation. These strategies (BPs, anti-RANKL: denosumab) could be proposed not only to target the bone component of the primary tumor but also to target bone/bone marrow metastases, the worst prognosis factor for Ewing sarcoma patients, as confirmed in the R3 arm of the latest EuroEWING99 trial (survival rate of $<20 \%$ at 5 years). However, as expected, these strategies have no effect in preclinical models of pulmonary metastases, which remains the main cause of mortality in Ewing sarcoma patients (the prognosis for patients with lung-only metastases is $30 \%$ survival at 5 years). However, the strategies could be proposed for pulmonary metastatic patients or patients with soft tissue Ewing sarcoma if they are in synergy with current or targeted therapies, as suggested by our preclinical studies combining ZOL with ifosfamide (65). 


\section{REFERENCES}

1. Ewing J. Diffuse endothelioma of bone. Proc N Y Pathol Soc (1921) 21:17-24.

2. Riggi N, Stamenkovic I. The biology of Ewing sarcoma. Cancer Lett (2007) 254:1-10. doi:10.1016/j.canlet.2006.12.009

3. Lau YS, Adamopoulos IE, Sabokbar A, Giele H, Gibbons CLMH, Athanasou NA. Cellular and humoral mechanisms of osteoclast formation in Ewing's sarcoma. Br J Cancer (2007) 96:1716-22. doi:10.1038/sj.bjc.6603774

4. Kovar H, Dworzak M, Strehl S, Schnell E, Ambros IM, Ambros PF, et al. Overexpression of the pseudoautosomal gene MIC2 in Ewing's sarcoma and peripheral primitive neuroectodermal tumor. Oncogene (1990) 5:1067-70.

5. Turc-Carel C, Philip I, Berger MP, Philip T, Lenoir G. Chromosomal translocation $(11 ; 22)$ in cell lines of Ewing's sarcoma. C R Seances Acad Sci III (1983) 296(23):1101-3.

6. Delattre O, Zucman J, Plougastel B, Desmaze C, Melot T, Peter M, et al. Gene fusion with an ETS DNA-binding domain caused by chromosome translocation in human tumours. Nature (1992) 359(6391):162-5. doi: $10.1038 / 359162 \mathrm{a} 0$

7. Arvand A, Denny CT. Biology of EWS/ETS fusions in Ewing's family tumors. Oncogene (2001) 20(40):5747-54. doi:10.1038/sj.onc.1204598

8. May WA, Gishizky ML, Lessnick SL, Lunsford LB, Lewis BC, Delattre O, et al. Ewing sarcoma 11;22 translocation produces a chimeric transcription factor that requires the DNA-binding domain encoded by FLI1 for transformation. Proc Natl Acad Sci U S A (1993) 90(12):5752-6. doi:10.1073/pnas.90.12.5752

9. Dauphinot L, De Oliveira C, Melot T, Sevenet N, Thomas V, Weissman BE, et al. Analysis of the expression of cell cycle regulators in Ewing cell lines: EWS-FLI-1 modulates p57KIP2and c-Myc expression. Oncogene (2001) 20(25):3258-65. doi:10.1038/sj.onc.1204437

10. Kovar H. Ewing's sarcoma and peripheral primitive neuroectodermal tumors after their genetic union. Curr Opin Oncol (1998) 10(4):334-42. doi:10.1097/00001622-199807000-00010

11. Erkizan HV, Uversky VN, Toretsky JA. Oncogenic partnerships: EWS-FLI1 protein interactions initiate key pathways of Ewing's sarcoma. Clin Cancer Res (2010) 16:4077-83. doi:10.1158/1078-0432.CCR-09-2261

12. Potratz J, Jürgens H, Craft A, Dirksen U. Ewing sarcoma: biology-based therapeutic perspectives. Pediatr Hematol Oncol (2012) 29:12-27. doi:10.3 109/08880018.2011.627582

13. Gaspar N, Hawkins DS, Dirksen U, Lewis IJ, Ferrari S, Le Deley MC, et al. Ewing sarcoma: current management and future approaches through collaboration. J Clin Oncol (2015) 27:3036-46. doi:10.1200/JCO.2014.59.5256

14. Iwasaki H, Suda T. Cancer stem cells and their niche. Cancer Sci (2009) 100:1166-72. doi:10.1111/j.1349-7006.2009.01177.x

15. Basak GW, Srivastava AS, Malhotra R, Carrier E. Multiple myeloma bone marrow niche. Curr Pharm Biotechnol (2009) 10:345-6.

16. Paget $\mathrm{S}$. The distribution of secondary growths in cancer of the breast. Lancet (1889) 133:571-3. doi:10.1016/S0140-6736(00)49915-0

17. Yin T, Li L. The stem cell niches in bone. J Clin Invest (2006) 116:1195-201. doi:10.1172/JCI28568

18. Hosokawa K, Arai F, Yoshihara H, Iwasaki H, Nakamura Y, Gomei Y, et al. Knockdown of N-cadherin suppresses the long-term engraftment of hematopoieticstem cells. Blood(2010) 116:554-63. doi:10.1182/blood-2009-05-224857

19. Clézardin P. Early bone metastasis-associated molecular and cellular events. In: Heymann D, editor. Bone Cancer. (Vol. 3), London, Burlington, San Diego: Academic Press is an imprint of Elsevier (2010). p. 41-5.

20. Coghlin C, Murray GI. Current and emerging concepts in tumour metastasis. J Pathol (2010) 222:1-15. doi:10.1002/path.2727

21. Mc Allister SS, Weinberg RA. Tumor-host interactions: a far-reaching relationship. J Clin Oncol (2010) 28:4022-8. doi:10.1200/JCO.2010.28.4257

22. Balkwill FR, Capasso M, Hagemann T. The tumor microenvironment at a glance. J Cell Sci (2012) 125:5591-6. doi:10.1242/jcs.116392

23. Fang H, DeClerck YA. Targeting the tumor microenvironment: from understanding pathways to effective clinical trials. Cancer Res (2013) 73:4965-77. doi:10.1158/0008-5472.CAN-13-0661

24. Miles FL, Sikes RA. Insidious changes in stromal matrix fuel cancer progression. Mol Cancer Res (2014) 12:297-312. doi:10.1158/1541-7786. MCR-13-0535

25. Paez D, Labonte MJ, Bohanes P, Zhang W, Benhanim L, Ning Y, et al. Cancer dormancy: a model of early dissemination and late cancer occurrence. Clin Cancer Res (2012) 18:645-53. doi:10.1158/1078-0432.CCR-11-2186
26. Theoleyre S, Wittrant Y, Tat SK, Fortun Y, Redini F, Heymann D. The molecular triad OPG/RANK/RANKL: involvement in the orchestration of pathophysiological bone remodeling. Cytokine Growth Factor Rev (2004) 15:457-75. doi:10.1016/j.cytogfr.2004.06.004

27. Wittrant Y, Théoleyre S, Chipoy C, Padrines M, Blanchard F, Heymann D, et al. RANKL/RANK/OPG: new therapeutic targets in bone tumours and associated osteolysis. Biochim Biophys Acta (2004) 1704:49-57.

28. David E, Blanchard F, Heymann M-F, De Pinieux G, Gouin F, Rédini F, et al. The bone niche of chondrosarcoma: a sanctuary for the drug resistance, the tumour growth and also a source of new therapeutic targets. Sarcoma (2011) 2011:1-8. doi:10.1155/2011/932451

29. Lacey DL, Timms E, Tan HL, Kelley MJ, Dunstan CR, Burgess T, et al. Osteoprotegerin ligand is a cytokine that regulates osteoclast differentiation and activation. Cell (1998) 93:165-76. doi:10.1016/S0092-8674(00)81569-X

30. Shiozawa Y, Eber MR, Berry JE, Taichman RS. Bone marrow as a metastatic niche for disseminated tumor cells from solid tumors. Bonekey Rep (2015) 4:689. doi:10.1038/bonekey.2015.57

31. Criscitiello C, Viale G, Gelao L, Esposito A, De Laurentiis M, De Placido S, et al. Crosstalk between bone niche and immune system: osteoimmunology signaling as a potential target for cancer treatment. Cancer Treat Rev (2015) 41:61-8. doi:10.1016/j.ctrv.2014.12.001

32. Chirgwin JM, Guise TA. Molecular mechanisms of tumor-bone interactions in osteolytic metastases. Crit Rev Eukaryot Gene Expr (2000) 10:159-78. doi:10.1615/CritRevEukarGeneExpr.v10.i2.50

33. Rogers MJ. New insights into the molecular mechanisms of action of bisphosphonates. Curr Pharm Des (2003) 9:2643-58. doi:10.2174/1381612033453640

34. Body JJ. Zoledronate. Cancer (1997) 80(Suppl 8):1699-701.

35. Cheer SM, Noble S. Zoledronic acid. Drugs (2001) 61:799-805. doi:10.2165/00003495-200161060-00010

36. Gatti D, Adami S. New bisphosphonates in the treatment of bone diseases. Drugs Aging (1999) 15:285-96. doi:10.2165/00002512-199915040-00004

37. Pataki A, Müller K, Green JR, Ma YF, Li QN, Jee WS. Effects of short-term treatment with the bisphosphonate zoledronate and pamidronate on rat bone: a comparative histomorphometric study on the cancellous bone formed before, during, and after treatment. Anat Rec (1997) 249:458-68. doi:10.1002/(SICI)1097-0185(199712)249:4<458::AID-AR5>3.0.CO;2-N

38. Epstein S. Update of current therapeutic options for the treatment of postmenopausal osteoporosis. Clin Ther (2006) 28:151-73. doi:10.1016/j. clinthera.2006.02.007

39. Heymann D, Ory B, Gouin F, Green JR, Rédini F. Bisphosphonates: new therapeutic agents for the treatment of bone tumors. Trends Mol Med (2004) 10:337-43. doi:10.1016/j.molmed.2004.05.007

40. Coleman RE. Risks and benefits of bisphosphonates. Br J Cancer (2008) 98:1736-40. doi:10.1038/sj.bjc.6604382

41. Dunford JE, Rogers MJ, Ebetino FH, Phipps RJ, Coxon FP. Inhibition of protein prenylation by bisphosphonates causes sustained activation of Rac, Cdc42, and Rho GTPases. J Bone Miner Res (2006) 21:684-94. doi:10.1359/ jbmr.060118

42. Coxon FP, Helfrich MH, Van't Hof R, Sebti S, Ralston SH, Hamilton A, et al. Protein geranylgeranylation is required for osteoclast formation, function, and survival: inhibition by bisphosphonates and GGTI-298. J Bone Miner Res (2000) 15:1467-76. doi:10.1359/jbmr.2000.15.8.1467

43. Benford HL, McGowan NW, Helfrich MH, Nuttall ME, Rogers MJ. Visualization of bisphosphonate-induced caspase-3 activity in apoptoticosteoclasts in vitro. Bone (2001) 28:465-73. doi:10.1016/S8756-3282(01)00412-4

44. Hughes DE, Wright KR, Uy HL, Sasaki A, Yoneda T, Roodman GD, et al. Bisphosphonates promote apoptosis in murine osteoclasts in vitro and in vivo. J Bone Miner Res (1995) 10:1478-87. doi:10.1002/jbmr.5650101008

45. Viereck V, Emons G, Lauck V, Frosch KH, Blaschke S, Gründker C, et al. Bisphosphonates pamidronate and zoledronic acid stimulate osteoprotegerin production by primary human osteoblasts. Biochem Biophys Res Commun (2002) 291:680-6. doi:10.1006/bbrc.2002.6510

46. Yoneda T, Hashimoto N, Hiraga T. Bisphosphonate actions on cancer. Calcif Tissue Int (2003) 73:315-8. doi:10.1007/s00223-002-0025-x

47. Watts NB, Diab DL. Long-term use of bisphosphonates in osteoporosis. JClin Endocrinol Metab (2010) 95:1555-65. doi:10.1210/jc.2009-1947

48. Brufsky AM. Zoledronic acid for cancer therapy - induced and postmenopausal bone loss. Expert Opin Pharmacother (2008) 9:1013-28. doi:10.1517/14656566.9.6.1013 
49. Jahanzeb M, Hirsh V. Bisphosphonates in lung cancer: more than a palliative therapy? Semin Oncol (2010) 37(Suppl 1):S45-52. doi:10.1053/j. seminoncol.2010.06.004

50. Boudou-Rouquette P, Alexandre J, Soubrane O, Bertagna X, Goldwasser F. Antitumoral effect of the bisphosphonate zoledronic acid against visceral metastases in an adrenocortical cancer patient. Ann Oncol (2009) 20:1747. doi:10.1093/annonc/mdp378

51. Kijima T, Fujii Y, Suyama T, Okubo Y, Yonese J, Fukui I. Lung and bone metastases from renal cell carcinoma responsive to bisphosphonates: a case report. Int J Urol (2008) 15:546-7. doi:10.1111/j.1442-2042.2008.02044.x

52. Diel IJ, Solomayer EF, Costa SD, Gollan C, Goerner R, Wallwiener D, et al. Reduction in new metastases in breast cancer with adjuvant clodronate treatment. NEngl J Med (1998) 339:357-63. doi:10.1056/NEJM199808063390601

53. Horie N, Murata H, Kimura S, Takeshita H, Sakabe T, Matsui T, et al. Combined effects of a third-generation bisphosphonate, zoledronic acid with other anticancer agents against murine osteosarcoma. Br J Cancer (2007) 9:255-61. doi:10.1038/sj.bjc.6603548

54. Ory B, Heymann M, Kamijo A, Gouin F, Heymann D, Rédini F. Zoledronic acid suppresses lung metastases and prolongs overall survival of osteosarcoma-bearing mice. Cancer (2005) 104:2522-9. doi:10.1002/cncr.21530

55. Koto K, Horie N, Kimura S, Murata H, Sakabe T, Matsui T, et al. Clinically relevant dose of zoledronic acid inhibits spontaneous lung metastasis in a murine osteosarcoma model. Cancer Lett (2009) 274:271-8. doi:10.1016/j. canlet.2008.09.026

56. Heymann D, Ory B, Blanchard F, Heymann MF, Coipeau P, Charrier C, et al. Enhanced tumor regression and tissue repair when zoledronic acid is combined with ifosfamide in rat osteosarcoma. Bone (2005) 37:74-86. doi:10.1016/j.bone.2005.02.020

57. Kubista B, Trieb K, Sevelda F, Toma C, Arrich F, Heffeter P, et al. Anticancer effects of zoledronic acid against human osteosarcoma cells. J Orthop Res (2006) 24:1145-52. doi:10.1002/jor.20129

58. Kubo T, Shimose S, Matsuo T, Sakai A, Ochi M. Efficacy of a nitrogen-containing bisphosphonate, minodronate, in conjunction with a p38 mitogen activated protein kinase inhibitor or doxorubicin against malignant bone tumor cells. Cancer Chemother Pharmacol (2008) 62:111-6. doi:10.1007/ s00280-007-0580-y

59. Benassi MS, Chiechi A, Ponticelli F, Pazzaglia L, Gamberi G, Zanella L, et al. Growth inhibition and sensitization to cisplatin by zoledronic acid in osteosarcoma cells. Cancer Lett (2007) 250:194-205. doi:10.1016/j. canlet.2006.10.004

60. Labrinidis A, Hay S, Liapis V, Ponomarev V, Findlay DM, Evdokiou A. Zoledronic acid inhibits both the osteolytic and osteoblastic components of osteosarcoma lesions in a mouse model. Clin Cancer Res (2009) 15:3451-61. doi:10.1158/1078-0432.CCR-08-1616

61. Meyers PA, Healey JH, Chou AJ, Wexler LH, Merola PR, Morris CD, et al. Addition of pamidronate to chemotherapy for the treatment of osteosarcoma. Cancer (2011) 117:1736-44. doi:10.1002/cncr.25744

62. Anderson P, Kopp L, Anderson N, Cornelius K, Herzog C, Hughes D, et al. Novel bone cancer drugs: investigational agents and control paradigms for primary bone sarcomas (Ewing's sarcoma and osteosarcoma). Expert Opin Investig Drugs (2008) 17:1703-15. doi:10.1517/13543784.17.11.1703

63. Sonnemann J, Eckervogt V, Truckenbrod B, Boos J, Winkelmann W, van Valen $\mathrm{F}$. The bisphosphonate pamidronate is a potent inhibitor of Ewing's sarcoma cell growth in vitro. Anticancer Drugs (2003) 14:767-71. doi:10.1097/00001813-200310000-00013

64. Zhou Z, Guan H, Duan X, Kleinerman ES. Zoledronic acid inhibits primary bone tumor growth in Ewing sarcoma. Cancer (2005) 104:1713-20. doi:10.1002/cncr.21383

65. Odri GA, Dumoucel S, Picarda G, Battaglia S, Lamoureux F, Corradini N, et al. Zoledronic acid as a new adjuvant therapeutic strategy for Ewing's sarcoma patients. Cancer Res (2010) 70:7610-9. doi:10.1158/0008-5472. CAN-09-4272

66. Murayama T, Kawasoe Y, Yamashita Y, Ueno Y, Minami S, Yokouchi M, et al. Efficacy of the third-generation bisphosphonate risedronate alone and in combination with anticancer drugs against osteosarcoma cell lines. Anticancer Res (2008) 28:2147-54.

67. Neville-Webbe HL, Evans CA, Coleman RE, Holen I. Mechanisms of the synergistic interaction between the bisphosphonate zoledronic acid and the chemotherapy agent paclitaxel in breast cancer cells in vitro. Tumour Biol (2006) 27:92-103. doi:10.1159/000092489

68. Yano S, Zhang H, Hanibuchi M, Miki T, Goto H, Uehara H, et al. Combined therapy with a new bisphosphonate, minodronate (YM529), and chemotherapy for multiple organ metastases of small cell lung cancer cells in severe combined immunodeficient mice. Clin Cancer Res (2003) 9:5380-5.

69. Odri G, Kim PP, Lamoureux F, Charrier C, Battaglia S, Amiaud J, et al. Zoledronic acid inhibits pulmonary metastasis dissemination in a preclinical model of Ewing's sarcoma via inhibition of cell migration. BMC Cancer (2014) 14:169. doi:10.1186/1471-2407-14-169

70. Goldsby RE, Fan TM, Villaluna D, Wagner LM, Isakoff MS, Meyer J, et al. Feasibility and dose discovery analysis of zoledronic acid with concurrent chemotherapy in the treatment of newly diagnosed metastatic osteosarcoma: a report from the Children's Oncology Group. Eur J Cancer (2013) 49:2384-91. doi:10.1016/j.ejca.2013.03.018

71. Battaglia S, Dumoucel S, Chesneau J, Heymann MF, Picarda G, Gouin F, et al. Impact of oncopediatric dosing regimen of zoledronic acid on bone growth: preclinical studies and case report of an osteosarcoma pediatric patient. $J$ Bone Miner Res (2011) 26:2439-51. doi:10.1002/jbmr.453

72. Lézot F, Chesneau J, Battaglia S, Brion R, Castaneda B, Farges JC, et al. Preclinical evidence of potential craniofacial adverse effect of zoledronic acid in pediatric patients with bone malignancies. Bone (2014) 68:146-52. doi:10.1016/j.bone.2014.08.018

73. Croucher PI, Shipman CM, Lippitt J, Perry M, Asosingh K, Hijzen A, et al. Osteoprotegerin inhibits the development of osteolytic bone disease in multiple myeloma. Blood (2001) 98:3534-40. doi:10.1182/blood.V98.13.3534

74. Zhang J, Dai J, Qi Y, Lin DL, Smith P, Strayhorn C, et al. Osteoprotegerin inhibits prostate cancer-induced osteoclastogenesis and prevents prostate tumor growth in the bone. J Clin Invest (2001) 107:1235-44. doi:10.1172/ JCI11685

75. Michigami T, Ihara-Watanabe M, Yamazaki M, Ozono K. Receptor activator of nuclear factor kappaB ligand (RANKL) is a key molecule of osteoclast formation for bone metastasis in a newly developed model of human neuroblastoma. Cancer Res (2001) 61:1637-44.

76. Mori K, Blanchard F, Charrier C, Battaglia S, Ando K, Duplomb L, et al. Conditioned media from mouse osteosarcoma cells promote MC3T3-E1 cell proliferation using JAKs and PI3-K/Akt signal crosstalk. Cancer Sci (2008) 99:2170-6. doi:10.1111/j.1349-7006.2008.00919.x

77. Wittrant Y, Lamoureux F, Mori K, Riet A, Kamijo A, Heymann D, et al. RANKL directly induces bone morphogenetic protein-2 expression in RANK-expressing POS-1 osteosarcoma cells. Int J Oncol (2006) 28:261-9.

78. Beristain AG, Narala SR, Di Grappa MA, KhoKha R. Homotypic RANKL signaling differentially regulates proliferation, motility and cell survival in osteosarcoma and mammary epithelial cells. J Cell Sci (2012) 125:943-55. doi: $10.1242 /$ jcs.094029

79. Rousseau J, Escriou V, Lamoureux F, Brion R, Chesneau J, Battaglia S, et al. Formulated siRNAs targeting Rankl prevent osteolysis and enhance chemotherapeutic response in osteosarcoma models. J Bone Miner Res (2011) 26:2452-62. doi:10.1002/jbmr.455

80. Redini F, Odri GA, Picarda G, Gaspar N, Heymann MF, Corradini N, et al. Drugs targeting the bone microenvironment: new therapeutic tools in Ewing's sarcoma? Expert Opin Emerg Drugs (2013) 18:339-52. doi:10.1517 /14728214.2013.823948

81. Lamoureux F, Richard P, Wittrant Y, Battaglia S, Pilet P, Trichet V, et al. Therapeutic relevance of osteoprotegerin gene therapy in osteosarcoma: blockade of the vicious cycle between tumor cell proliferation and bone resorption. Cancer Res (2007) 67:7308-18. doi:10.1158/0008-5472. CAN-06-4130

82. Lamoureux F, Picarda G, Rousseau J, Gourden C, Battaglia S, Charrier C, et al. Therapeutic efficacy of soluble receptor activator of nuclear factor-kappa $\mathrm{B}-\mathrm{Fc}$ delivered by nonviral gene transfer in a mouse model of osteolytic osteosarcoma. Mol Cancer Ther (2008) 7:3389-98. doi:10.1158/1535-7163. MCT-08-0497

83. Simonet WS, Lacey DL, Dunstan CR, Kelley M, Chang MS, Lüthy R, et al. Osteoprotegerin: a novel secreted protein involved in the regulation of bone density. Cell (1997) 89:309-19. doi:10.1016/S0092-8674(00)80209-3

84. Hofbauer LC, Khosla S, Dunstan CR, Lacey DL, Boyle WJ, Riggs BL. The roles of osteoprotegerin and osteoprotegerin ligand in the paracrine 
regulation of bone resorption. J Bone Miner Res (2000) 15:2-12. doi:10.1359/ jbmr.2000.15.1.2

85. Yasuda H, Shima N, Nakagawa N, Mochizuki SI, Yano K, Fujise N, et al. Identity of osteoclastogenesis inhibitory factor (OCIF) and osteoprotegerin (OPG): a mechanism by which OPG/OCIF inhibits osteoclastogenesis in vitro. Endocrinology (1998) 139:1329-37. doi:10.1210/endo.139.3.5837

86. Grimaud E, Soubigou L, Couillaud S, Coipeau P, Moreau A, Passuti N, et al. Receptor activator of nuclear factor kappaB ligand (RANKL)/osteoprotegerin (OPG) ratio is increased in severe osteolysis. Am J Pathol (2003) 163:2021-31. doi:10.1016/S0002-9440(10)63560-2

87. Emery JG, McDonnell P, Burke MB, Deen KC, Lyn S, Silverman C, et al. Osteoprotegerin is a receptor for the cytotoxic ligand TRAIL. J Biol Chem (1998) 273:14363-7. doi:10.1074/jbc.273.23.14363

88. Lamoureux F, Moriceau G, Picarda G, Rousseau J, Trichet V, Rédini F. Regulation of osteoprotegerin pro- or anti-tumoral activity by bone tumor microenvironment. Biochim Biophys Acta (2010) 1805:17-24. doi:10.1016/j. bbcan.2009.08.004

89. Picarda G, Lamoureux F, Geffroy L, Delepine P, Montier T, Laud K, et al. Preclinical evidence that use of TRAIL in Ewing's sarcoma and osteosarcoma therapy inhibits tumor growth, prevents osteolysis, and increases animal survival. Clin Cancer Res (2010) 16:2363-74. doi:10.1158/1078-0432. CCR-09-1779

90. Abadie A, Wietzerbin J. Involvement of TNF-related apoptosis-inducing ligand (TRAIL) induction in interferon gamma-mediated apoptosis in Ewing tumor cells. Ann N Y Acad Sci (2003) 10:10117-20.

91. Virk MS, Alaee F, Petrigliano FA, Sugiyama O, Chatziioannou AF, Stout D, et al. Combined inhibition of the BMP pathway and the RANK-RANKL axis in a mixed lytic/blastic prostate cancer lesion. Bone (2011) 48:578-87. doi:10.1016/j.bone.2010.11.003

92. Feeley BT, Liu NQ, Conduah AH, Krenek L, Roth K, Dougall WC, et al. Mixed metastatic lung cancer lesions in bone are inhibited by noggin overexpression and Rank:Fc administration. J Bone Miner Res (2006) 21:1571-80. doi:10.1359/jbmr.060706

93. Holland PM, Miller R, Jones J, Douangpanya H, Piasecki J, Roudier M, et al. Combined therapy with the RANKL inhibitor RANK-Fc and rhApo2L/ TRAIL/dulanermin reduces bone lesions and skeletal tumor burden in a model of breast cancer skeletal metastasis. Cancer Biol Ther (2010) 9:539-50. doi:10.4161/cbt.9.7.11266

94. McClung MR, Lewiecki EM, Cohen SB, Bolognese MA, Woodson GC, Moffett $\mathrm{AH}$, et al. Denosumab in postmenopausal women with low bone mineral density. N Engl J Med (2006) 354:821-31. doi:10.1056/NEJMoa044459

95. Body JJ, Facon T, Coleman RE, Lipton A, Geurs F, Fan M, et al. A study of the biological receptor of nuclear factor-kappaB ligand inhibitor, denosumab, in patients with multiple myeloma or bone metastases from breast cancer. Clin Cancer Res (2006) 12:1221-8. doi:10.1158/1078-0432.CCR-05-1933

96. Fizazi K, Carducci M, Smith M, Damião R, Brown J, Karsh L, et al. Denosumab versus zoledronic acid for treatment of bone metastases in men with castration-resistant prostate cancer: a randomised, double-blind study. Lancet (2011) 377:813-22. doi:10.1016/S0140-6736(10)62344-6

97. Henry DH, Costa L, Goldwasser F, Hirsh V, Hungria V, Prausova J, et al. Randomized, double-blind study of denosumab versus zoledronic acid in the treatment of bone metastases in patients with advanced cancer (excluding breast and prostate cancer) or multiple myeloma. J Clin Oncol (2011) 29:1125-32. doi:10.1200/JCO.2010.31.3304

98. Stopeck AT, Lipton A, Body JJ, Steger GG, Tonkin K, de Boer RH, et al. Denosumab compared with zoledronic acid for the treatment of bone metastases in patients with advanced cancer: a randomized, double-blind study. J Clin Oncol (2010) 28:5132-9. doi:10.1200/JCO.2010.29.7101

99. Thomas D, Henshaw R, Skubitz K, Chawla S, Staddon A, Blay JY, et al. Denosumab in patients with giant-cell tumour of bone: an open-label, phase 2 study. Lancet Oncol (2010) 11:275-80. doi:10.1016/S1470-2045(10)70010-3

100. Semenza GL. The hypoxic tumor microenvironment: a driving force for breast cancer progression. Biochim Biophys Acta (2015). doi:10.1016/j. bbamcr.2015.05.036

101. Dunst J, Ahrens S, Paulussen M, Burdach S, Jürgens H. Prognostic impact of tumor perfusion in MR-imaging studies in Ewing tumors. Strahlenther Onkol (2001) 177:153-9.
102. Aryee DN, Niedan S, Kauer M, Schwentner R, Bennani-Baiti IM, Ban J, et al. Hypoxia modulates EWS-FLI1 transcriptional signature and enhances the malignant properties of Ewing's sarcoma cells in vitro. Cancer Res (2010) 70:4015-23. doi:10.1158/1538-7445.AM10-444

103. Kauer M, Ban J, Kofler R, Walker B, Davis S, Meltzer P, et al. A molecular function map of Ewing's sarcoma. PLoS One (2009) 4:e5415. doi:10.1371/ journal.pone. 0005415

104. Berghuis D, Santos SJ, Baelde HJ, Taminiau AH, Egeler RM, Schilham MW, et al. Pro-inflammatory chemokine-chemokine receptor interactions within the Ewing sarcoma microenvironment determine CD8(+) T-lymphocyte infiltration and affect tumor progression. J Pathol (2011) 223:347-57. doi:10.1002/path.2819

105. Reddy K, Cao Y, Zhou Z, Yu L, Jia SF, Kleinerman ES. VEGF165 expression in the tumor microenvironment influences the differentiation of bone marrow-derived pericytes that contribute to the Ewing's sarcoma vasculature. Angiogenesis (2008) 11:257-67. doi:10.1007/s10456-008-9109-1

106. Guan H, Zhou Z, Cao Y, Duan X, Kleinerman ES. VEGF165 promotes the osteolytic bone destruction of Ewing's sarcoma tumors by upregulating RANKL. Oncol Res (2009) 18:117-25. doi:10.3727/096504009789954627

107. Kamura S, Matsumoto Y, Fukushi JI, Fujiwara T, Iida K, Okada Y, et al. Basic fibroblast growth factor in the bone microenvironment enhances cell motility and invasion of Ewing's sarcoma family of tumours by activating the FGFR-PI3K-Racl pathway. Br J Cancer (2010) 103:370-81. doi:10.1038/ sj.bjc. 6605775

108. Haider MT, Holen I, Dear TN, Hunter K, Brown HK. Modifying the osteoblastic niche with zoledronic acid in vivo-potential implications for breast cancer. Bone (2014) 66:240-50. doi:10.1016/j.bone.2014.06.023

109. Olechnowicz SW, Edwards CM. Contribution of the host microenvironment to cancer-induced bone disease. Cancer Res (2014) 74:1625-31. doi:10.1158/0008-5472.CAN-13-2645

110. Weilbaecher KN, Guise TA, McCauley LK. Cancer to bone: a fatal attraction. Nat Rev Cancer (2011) 11:411-25. doi:10.1038/nrc3055

111. Fong EL, Lamhamedi-Cherradi SE, Burdett E, Ramamoorthy V, Lazar AJ, Kasper FK, et al. Modeling Ewing sarcoma tumors in vitro with 3D scaffolds. Proc Natl Acad Sci U S A (2013) 110:6500-5. doi:10.1073/pnas.1221403110

112. Lamhamedi-Cherradi S-E, Santoro M, Ramammoorthy V, Menegaz BA, Bartholomeusz G, Iles LR, et al. 3D tissue-engineered model of Ewing's sarcoma. Adv Drug Del Rev (2014) 7(9-80):155-71. doi:10.1016/j. addr.2014.07.012

113. Villasante A, Marturano-Kruik A, Vunjak-Novakovic G. Bioengineered human tumor within a bone niche. Biomaterials (2014) 35:5785-94. doi:10.1016/j.biomaterials.2014.03.081

114. Dalal S, Burchill SA. Preclinical evaluation of vascular disrupting agents in Ewing's sarcoma family of tumours. Eur J Cancer (2009) 45:713-22. doi:10.1016/j.ejca.2008.11.045

115. Vormoor J, Baersch G, Decker S, Hotfilder M, Schäfer KL, Pelken L, et al. Establishment of an in vivo model for pediatric Ewing tumours by transplantation into NOD/SCID mice. Pediatr Res (2001) 49:332-41. doi:10.1203/00006450-200103000-00006

116. Mazurier F, Doedens M, Gan OI, Dick JE. Rapid myeloerythroid repopulation after intrafemoral transplantation in NOD-SCID mice reveals a new class of human stem cells. Nat Med (2003) 9:959-63. doi:10.1038/nm886

117. Vormoor B, Knizia HK, Batey MA, Almeida GS, Wilson I, Dildey P, et al. Development of a preclinical orthotopic xenograft model of Ewing sarcoma and other human malignant bone disease using advanced in vivo imaging. PLoS One (2014) 9(1):e85128. doi:10.1371/journal.pone.0085128

Conflict of Interest Statement: The authors declare that the research was conducted in the absence of any commercial or financial relationships that could be construed as a potential conflict of interest.

Copyright $\odot 2015$ Redini and Heymann. This is an open-access article distributed under the terms of the Creative Commons Attribution License (CC BY). The use, distribution or reproduction in other forums is permitted, provided the original author(s) or licensor are credited and that the original publication in this journal is cited, in accordance with accepted academic practice. No use, distribution or reproduction is permitted which does not comply with these terms. 